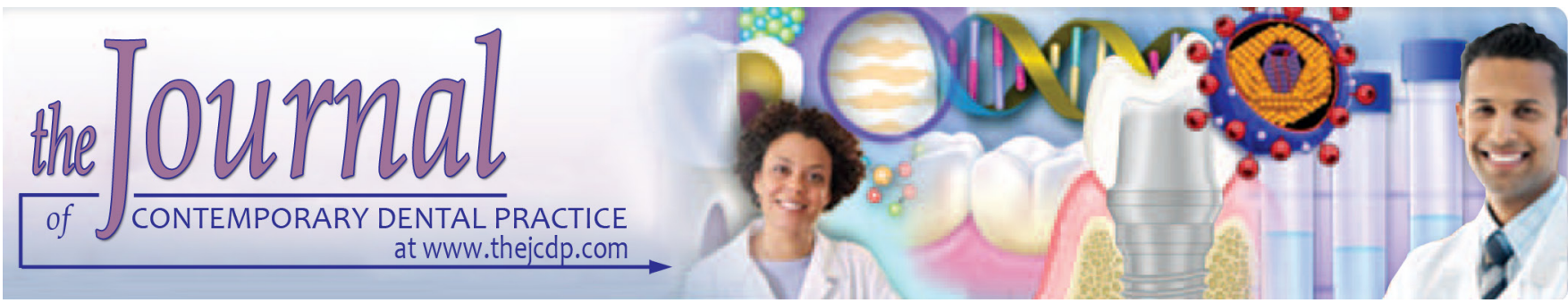

\title{
Evaluation of Effect of Glucosamine-Chondroitin Sulfate, Tramadol, and Sodium Hyaluronic Acid on Expression of Cytokine Levels in Internal Derangement of Temporomandibular Joint
}

\author{
${ }^{1}$ Srinivas Ganti, ${ }^{2}$ Prashant Shriram, ${ }^{3}$ Arsalan SA Ansari, ${ }^{4}$ Junaid MH Kapadia, ${ }^{5}$ Anurag Azad, ${ }^{6}$ Abhinav Dubey
}

\begin{abstract}
Aim: Evaluation of the effect of glucosamine-chondroitin combination, tramadol, and sodium hyaluronic acid in temporomandibular joint (TMJ) disorders and its impact on the expression of various cytokines such as IL-6, IL-1ß, TNF- $\alpha$, and PGE2.
\end{abstract}

Materials and methods: The present study was conducted on 60 patients (males-30, females-30) suffering from internal derangement such as disc displacement with reduction of TMJ. The patients were divided into three groups of 20 each. Group I received a combination of $1.5 \mathrm{~g}$ of glucosamine and $1.2 \mathrm{~g}$ of chondroitin sulfate per day and group II received $50 \mathrm{mg}$ tramadol HCL peroral. Group III received sodium hyaluronate 10 $\mathrm{mg} / \mathrm{mL}, 2 \mathrm{~mL}$ injection syringe on each joint. Pain (VAS) scale and maximum mouth opening (MMO) was measured. The level of IL-6, IL-1ß, TNF- $\alpha$, and PGE2 levels were measured using Enzyme-linked immuno sorbent assay (ELISA).

Results: There was an improvement in maximum mouth opening in all three groups $(p<0.05)$. There was a reduction in pain in all groups. IL- $1 ß$, TNF- $\alpha$, and PGE2 leve ls showed reduction while IL-6 showed an increase in value in group II and III.

Conclusion: The efficacy of glucosamine chondroitin sulfate, tramadol and hyaluronic acid in TMJ disorders has been found to be effective.

Clinical significance: IL-6, IL-1ß, TNF- $\alpha$, and PGE2 levels indicate the risk of TMJ disorders. Thus earlier assessment

\footnotetext{
${ }^{1,5,6}$ Deptartment of Oral and Maxillofacial Surgery, Bhabha College of Dental Sciences, Bhopal, Madhya Pradesh, India

${ }^{2}$ Deptartment of Oral and Maxillofacial Surgery, ACPM Dental College, Dhule, Maharashtra, India

${ }^{3}$ Deptartment of Oral and Maxillofacial Surgery, Terna Dental College, Navi Mumbai, Maharashtra, India

${ }^{4}$ Deptartment of Public Health Dentistry, Bhabha College of Dental Sciences, Bhopal, Madhya Pradesh, India

Corresponding Author: Junaid M Kapadia, Deptartment of Public Health Dentistry, Bhabha College of Dental Sciences, Bhopal, Madhya Pradesh, India, Phone: +917303320242, e-mail: drjunaid.kapadia@gmail.com
}

of their levels helps in diagnosis, and better management may be done.

Keywords: Chondroitin sulfate, Glucosamine, TMJ, Tramadol

How to cite this article: Ganti S, Shriram P, Ansari ASA, Kapadia JMH, Azad A, Dubey A. Evaluation of Effect of Glucosamine-Chondroitin Sulfate, Tramadol, and Sodium Hyaluronic Acid on Expression of Cytokine Levels in Internal Derangement of Temporomandibular Joint. J Contemp Dent Pract 2018;19(12):1502-1506.

Source of support: Nil

Conflict of interest: None

\section{INTRODUCTION}

Temporomandibular joint (TMJ) disorders are increasing in all age groups. It has become the common disorders affecting daily activities such as talking, eating and swallowing, etc. Various etiologies have been postulated causing TMJ disorders. It is considered to be multifactorial. These disorders may be noninflammatory or inflammatory. ${ }^{1}$ Among these, anterior disc displacement with or without reduction and osteoarthritis are common. Precipitating factors such as stress, parafunctional habits, anxiety, and occlusal disharmony are thought to be associated with it. The patient usually encounters pain in joints, limited mouth opening, clicking sound or crepitus while opening and closing mouth, headache and pain in the neck. ${ }^{2}$

Careful assessment of TMJ is necessary to determine disorder. TMJ palpation and muscle evaluation of masseter and sternocleidomastoid (SCM) muscle help in diagnosis. Trigger points on muscles can be elicited by digital palpation. ${ }^{3}$

It has been observed that there is the release of numerous inflammatory mediators such as interleukin 6 (IL-6), interleukin one beta (IL-1ß), tumor necrosis factor-alpha 
(TNF- $\alpha$ ) and prostaglandin E2 (PGE2) levels in joint space. Subsequently, there is a reduction in the secretion of proteoglycans in these disorders. Both $g$ lucosamine and chondroitin are proteinaceous, are component of cartilage. ${ }^{4}$

Several modalities such as the use of non-steroidal antiinflammatory drugs (NSAIDS), tricyclic antidepressants (TCA), corticosteroids or hyaluronate can help with acute and chronic arthritis pain have been tried. Muscle relaxants and benzodiazepines have been used as conservative treatment modalities. Tramadol is a nonanti-inflammatory drug with central activity. It is narcotic in nature. ${ }^{5}$ The present study was conducted to compare the efficacy of the combination of glucosamine chondroitin sulfate, tramadol and sodium hyaluronate in TMJ disorders. It also aimed to evaluate the alteration in the expression of various cytokine s such as IL-6, IL-1ß, TNF- $\alpha$, and PGE2.

\section{MATERIALS AND METHODS}

The present study was conducted on 60 subjects of internal derangement such as disc displacement with reduction of both genders (males-30, females-30). The diagnosis was made based on clinical examination and with magnetic resonance imaging (MRI). All were informed regarding the study, and written consent was obtained. Ethical clearance was obtained from the Institution Ethical Committee prior to the study.

General information such as name, age, gender etc. was recorded. The pain was measured on a VAS scale, and maximum mouth opening (MMO) was measured by calculating interincisal distance. Conscious sedation was performed by injecting $(0.1 \mathrm{mg} / \mathrm{kg})$ intravenous midazolam. All were administered auriculotemporal nerve block. Normal saline lavage was performed in upper joint space at a distance of $1 \mathrm{~cm}$ anterior to the tragus. This procedure was repeated to get a synovial fluid sample which was stored at $-70^{\circ} \mathrm{C}$. This sample was used to assess the level of IL-6, IL-1ß, TNF- $\alpha$, and PGE2.

The patients were divided into three groups of 20 each. Group I received a combination of $1.5 \mathrm{~g}$ of glucosamine and $1.2 \mathrm{~g}$ of chondroitin sulfate per day and group II received $50 \mathrm{mg}$ tramadol HCL peroral. Group III received Sodium hyaluronate $10 \mathrm{mg} / \mathrm{ml}, 2 \mathrm{ml}$ injection syringe on each joint. The same procedure was repeated after 8 weeks to obtain synovial fluid. There was no control group in the study. The level of IL-6, IL-1ß, TNF- $\alpha$, and PGE2 levels were measured using enzyme-linked immuno sorbent assay (ELISA). Results thus obtained were subjected to statistical analysis using chi-square and Wilcoxon signed rank test. A p-value of less than 0.05 was considered significant.

\section{RESULTS}

Pain VAS score was $7.12 \pm 2.8$ before and $3.20 \pm 1.5$ after treatment in group I. In group II, pain VAS score was 7.28 \pm 2.5 before and $3.25 \pm 2.2$ after treatment. The difference was significant in both groups ( $\mathrm{p}<0.05)$. Maximum mouth opening $(\mathrm{MMO})$ was $27.56 \pm 4.6 \mathrm{~mm}$ and $32.24 \pm$ $5.2 \mathrm{~mm}$ before and after treatment in group I whereas it was $26.14 \pm 3.8 \mathrm{~mm}$ and $30.14 \pm 4.4 \mathrm{~mm}$ before and after treatment in group II. The difference was significant in both groups $(\mathrm{p}<0.05)$. In group III, the pain score was 7.55 \pm 3.4 and $3.38 \pm 2.7$ before and after treatment and $\mathrm{MMO}$ was $28.17 \pm 3.2$ and $31.72 \pm 5.1$ before and after treatment. The difference was significant $(\mathrm{p}<0.05)$ (Table 1).

In group I, IL-1 1 level (pg/mL) was 42 before and 30 after treatment, IL- 6 was 51 and 39 before and after treatment, TNF- $\alpha$ level was 7.8 and 4.2 before and after treatment, and the PGE2 level was 7.4 and 6.3 before and after treatment respectively. The difference was significant except TNF- $\alpha$ and PGE2 level ( $p$ >0.05). (Graph 1).

In group II, IL-1ß level (pg/mL) was 43 before and 51 after treatment, IL-6 was 31 and 38 before and after treatment, TNF- $\alpha$ level was 7.56 and 7.18 before and after treatment, and the PGE2 level was 6.89 and 6.52 before

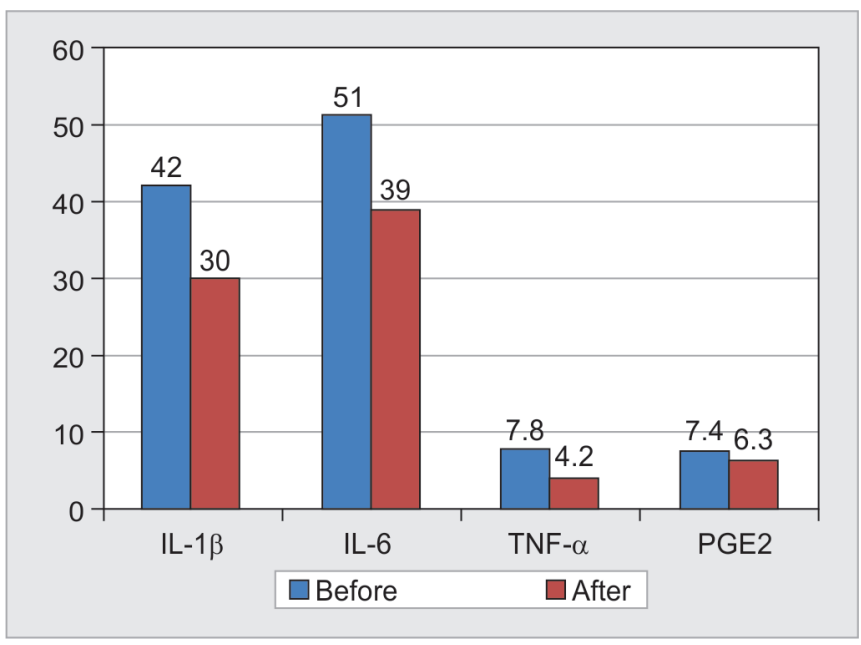

Graph 1: Measurement of IL- 1 ß, IL-6, TNF- $\alpha$ and PGE2 levels in group I

Table 1: Pain and MMO in both groups

\begin{tabular}{|c|c|c|c|c|c|c|c|c|c|}
\hline \multirow{2}{*}{$\begin{array}{l}\text { Para } \\
\text { meters }\end{array}$} & \multicolumn{2}{|c|}{ Group I } & \multirow[b]{2}{*}{$p$-value } & \multicolumn{2}{|c|}{ Group II } & \multirow[b]{2}{*}{$p$-value } & \multicolumn{2}{|c|}{ Group III } & \multirow[b]{2}{*}{$p$-value } \\
\hline & Before & After & & Before & After & & Before & After & \\
\hline Pain (VAS) & $7.12 \pm 2.8$ & $3.20 \pm 1.5$ & 0.01 & $7.28 \pm 2.5$ & $3.25 \pm 2.2$ & 0.02 & $7.55 \pm 3.4$ & $3.38 \pm 2.7$ & 0.01 \\
\hline $\mathrm{MMO}(\mathrm{mm})$ & $27.56 \pm 4.6$ & $32.24 \pm 5.2$ & 0.05 & $26.14 \pm 3.8$ & $30.14 \pm 4.4$ & 0.04 & $28.17 \pm 3.2$ & $31.72 \pm 5.1$ & 0.02 \\
\hline
\end{tabular}


and after treatment respectively. The difference was nonsignificant $(p>0.05)$ except IL-1ß level which showed a significant difference $(\mathrm{p}<0.05)$. (Graph 2$)$

In group III, IL-1ß level (pg/mL) was 45 before and 42 after treatment, IL- 6 was 32 and 39 before and after treatment, TNF- $\alpha$ level was 7.44 and 7.28 before and after treatment, and the PG E2 level was 6.76 and 6.41 before and after treatment respectively. The difference was nonsignificant ( $p>0.05)$ (Graph 3).

\section{DISCUSSION}

Temporomandibular Joint disorders are evident in the massive scale of the population worldwide. Among inflammatory disorders, internal derangement such as disc displacement with or without reduction is commonly encountered.6 Disc displacement with reduction is characterized by clicking sound while opening and closing mouth due to the displacement of a disc. However in disc displacement without reduction cases, there is locking of joint. The condition is so painful that the patient avoids opening the mouth.7 Excessive mouth opening as in yawning may initiate the pain process. These inflammatory disorders lead to alteration in cytokines levels such as IL-1 i level, IL- 6, TNF- , and PGE2 level. Chou et al.8 assessed MMP-9 and IL-1ß level on the administration of chondroitin and glucosamine sulfate for 25 days. The author found that chondroitin and glucosamine combination significantly decreased IL-1ß level. The MMP-9 level was elevated following administration of chondroitin sulfate. Thus both chondroitin and glucosamine in combination proved to be useful in arthritis.

In the present study, 60 patients diagnosed with internal derangement such as disc displacement with or without reduction were given glucosamine and chondroitin sulfate, tramadol and sodium hyaluronate in group I, II and III respectively. In all patients,

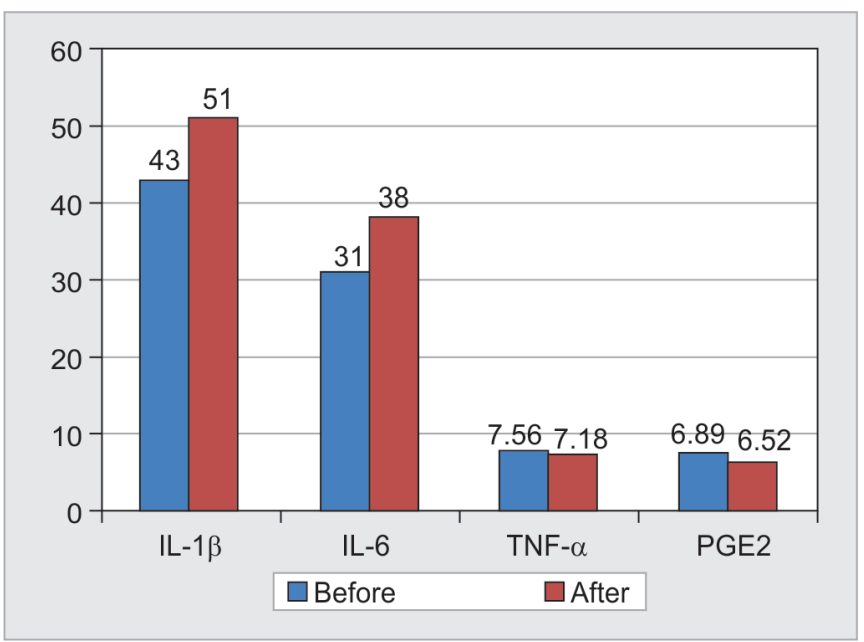

Graph 2: Measurement of IL- 1 ß, IL-6, TNF- $\alpha$ and PGE2 levels in group II arthrocentesis was done with sterile saline, and synovial fluid was obtained. It was observed that pain (VAS) score was reduced significantly in all groups. The decrease was more in group I as compared to group II and group III. There was more reduction in pain score in group I whereas group II and group III had slightly less decrease in VAS score.

Similarly, MMO was measured before and after treatment in all groups which revealed that group I had a higher value of mouth opening as compared to other groups. Mouth opening was measured by calculating the distance between the upper and lower incisal edges which was expressed in $\mathrm{mm}$. This is in agreement with Bouloux GF.'

In a study by Kaneyama et al. ${ }^{10} \mathrm{IL}-1 B$, IL-6, and $\mathrm{TNF}-\alpha$ levels were measured in synovial fluid of 55 osteoarthritides and five healthy subjects. The measurement of all inflammatory mediators showed higher values in all 55 osteoarthritis patients whereas healthy subjects showed no increase in level.

Hyaluronic acid (HA) is (HMW) a polysaccharide which is a normal component of the joint synovial fluid. Its use in TMJ osteoarthritis has shown improvement in signs and symptoms. The administration of low doses allows prolonging stay in joint space which initiates a therapeutic response. ${ }^{11}$ Glucosamine and chondroitin sulfate in the management of TMJ disorders may be useful. Nguyen et al. ${ }^{12}$ in their study involved 45 subjects who were given Glucosamine HCL and chondroitin sulfate. There was a decrease in pain value in patients with disc displacement, osteoarthritis, and capsulitis. The author found a reduction in clicking and crepitus in both TMJ hence advocated the use of these agents in various TMJ anomalies.

The use of tramadol in TMJ disorders has not done so far. We assessed the role of tramadol in relieving pain and

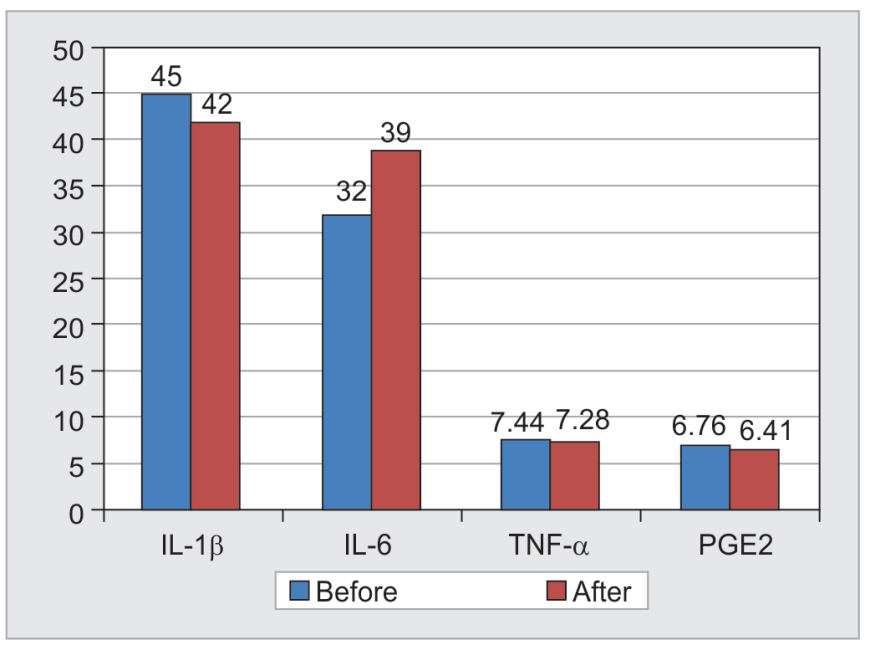

Graph 3: Measurement of IL- $1 ß$, IL-6, TNF- $\alpha$ and PGE2 levels in group III 
increasing mouth opening in patients. The results showed improvement in all factors. Its efficiency is equivalent to morphine. It is useful in both acute and chronic pain. Its use in controlling pain started long back, and for the last four decades it has been the choice of drug for controlling severe painful disorders. ${ }^{13}$

It was observed that IL-1ß level $(\mathrm{pg} / \mathrm{mL})$ was decreased in group I and III whereas it was slightly increased in group II. Similarly, the level of TNF- $\alpha$ and PGE2 showed a reduction in all groups whereas IL-6 level was increased in group II and III but in group I, the level showed a significant reduction. The increased level of IL-1ß and IL-6 in group II is due to poor antiinflammatory action of tramadol.

Shankland et al. ${ }^{14}$ in their study evaluated the role of glucosamine and chondroitin sulfate on osteoarthritis patients and observed that clicking sound and pain was reduced significantly. Quinn and Bazan $^{15}$ in their study showed that there is the release of inflammatory mediators such as PGE2 in synovial fluid of TMJ disorders such as internal derangements. In the present study, the PGE2 level was decreased owing to anti-inflammatory role of glucosamine-chondroitin sulfate which causes a reduction in its secretion and thus relieving pain and increasing mo uth opening. Glucosamine-chondroitin sulfate has been widely used in TMJ disorders such as arthritis and internal derangement. This combination has shown a reduction in cartilage breakdown. Burch et al. ${ }^{16}$ in a group of patients suffering from TMJ disorders compared placebo with tramadol and found that there was the significant reduction in pain on administration of $200 \mathrm{mg}$ drug daily.

Gencer et al. $^{17}$ assessed the role of hyaluronic acid, tenoxicam, and betametha sone on temporomandibular joint disorders such as disc displacement and osteoarthritis. Intraarticular injection of hyaluronic acid found efficient in controlling pain as compared to tenoxicam and betametha sone. The author concluded that hyaluronic acid is a useful medication for relieving pain and other symptoms in these disorders. Glucosamine chondroitin sulfate, tramadol and hyaluronic acid in TMJ disorders show improvement in mouth opening and reduction in pain values. Joint lavage may be useful in reducing pain in patients.

IL-6, IL-1ß, TNF- $\alpha$, and PGE2 are mediators of inflammation. Their level increases in TMJ disorders such as arthritis, internal derangements, etc. The present study assessed the role of glucosamine chondroitin sulfate, tramadol and hyaluronic acid on the secretion of these mediators. The limitation of the study is that a small sample size was considered. Drugs such as betamethasone and other corticosteroids were not considered.

\section{CONCLUSION}

The efficacy of glucosamine chondroitin sulfate, tramadol and hyaluronic acid in TMJ disorders has been found effective. There was a reduction in pain and an increase in mouth opening. There was an alteration in inflammatory mediators such as IL-6, IL-1ß, TNF- $\alpha$ and PGE2 levels.

\section{REFERENCES}

1. Kostrzewa-Janicka J, Mierzwinska-Nastalska E, Jurkowski P, Okonski P, Nedzi-Gora M. Assessment of temporomandibular joint disease. Adv Exp Med Biol. 2013; 788:207-211.

2. Kacena MA, Merrel GA, Konda SR, Wilson KM, Xi Y, Horowitz MC. Inflammation and bony changes at the temporomandibular joint. Cells Tissues Organs. 2001; 169:257-264.

3. Matsumoto K, Honda K, Ohshima M, Yamaguchi Y, Nakajima I, Micke P, et al. Cytokine profile in synovial fluid from patients with internal derangement of the temporomandibular joint: A preliminary study. Dentomaxillofac Radiol. 2006;35:432-441.

4. Wake M, Hamada Y, Kumagai K, Tanaka N, Ikeda Y, Nakatani $Y$, et al. Up-regulation of interleukin- 6 and vascular endothelial growth factor-A in the synovial fluid of temporomandibular joints affected by synovial chondromatosis. Br J Oral Maxillofac Surg. 2013;51:164-169.

5. Takano H, Ariyoshi W, Kanno T, Fukuhara E, Ichimiya $\mathrm{H}$, Matayoshi $\mathrm{T}$, et al. Induction of osteoclast-like cells derived from the synovial lavage fluids of patients with temporomandibular joint disorders. Osteoarthritis Cartilage. 2007;15:291-299.

6. Bruyere O, Reginster JY. Glucosamine and chondroitin sulfate as therapeutic agents for knee and hip osteoarthritis. Drugs Aging 2007;24:573-580.

7. Chan PS, Caron JP, Rosa GJ, Orth MW. Glucosamine and chondroitin sulfate regulate gene expression and synthesis of nitric oxide and prostaglandin E (2) in articular cartilage explants. Osteoarthritis Cartilage. 2005;13:387-394.

8. Bouloux GF. Temporomandibular joint pain and synovial fluid analysis: a review of the literature. J Oral Maxillofac Surg 2009;67:2497-504.

9. Chou MM, Vergnolle N, McDougall JJ, Wallace JL, Marty $\mathrm{S}$, Teskey $\mathrm{V}$ et al. Effects of chondroitin and glucosamine sulfate in a dietary bar formulation on inflammation, interleukin-1beta, matrix metalloprotease-9, and cartilage damage in arthritis. Exp Biol Med (Maywood). 2005; 230(4):255-262.

10. Kaneyama K, Segami N, Nishimura M, Suzuki T, Sato J. Importance of proinflammatory cytokines in synovial fluid from 121 joints with temporomandibular disorders. Br J Oral Maxillofac Surg. 2002;40:418-423.

11. Kean WF, Bouchard S, Roderich Gossen E. Women with pain due to osteoarthritis: the efficacy and safety of a oncedaily formulation of tramadol. Pain Med. 2009;10:1001-1011.

12. Nguyen P, Mohamed SE, Gardiner D, Salinas T. A randomized double-blind clinical trial of the effect of chondroitin sulfate and glucosamine hydrochloride on temporomandibular joint disorders: A pilot study. Cranio. 2001;19:130-139.

13. Mazieres B, Hucher M, Zaim M, Garnero P. Effect of chondroitin sulphate in symptomatic knee osteoarthritis: a 
multicentre, randomised, double-blind, placebo-controlled study. Ann Rheum Dis. 2007;66:639-645.

14. Shankland WE, 2nd. The effects of glucosamine and chondroitin sulfate on osteoarthritis of the TMJ: a preliminary report of 50 patients. Cranio. 1998;16:230-235.

15. Quinn JH, Bazan NG. Identification of prostaglandin E2 and leukotrienes B4 in the synovial fluid of painful, dysfunctional temporomandibular joints. J Oral Maxillofac Surg. 1990;48:968-971.
16. Burch F, Fishman R, Messina N, Corser B, Radulescu F, Sarbu A, et al. A comparison of the analgesic efficacy of Tramadol Contramid OAD versus placebo in patients with pain due to osteoarthritis. J Pain Symptom Manage. 2007; 34:328-338.

17. Gencer ZK, Özkiriş M, Okur A, Korkmaz M, Saydam L. A comparative study on the impact of intra-articular injections of hyaluronic acid, tenoxicam and betametasone on the relief of temporomandibular joint disorder complaints. Journal of Cranio-Maxillofacial Surgery. 2014;42(7):1117-1121. 